\title{
Computing Emotion Awareness Through Facial Electromyography
}

\author{
Egon L. van den Broek ${ }^{1,2}$, Marleen H. Schut ${ }^{2,3}$, Joyce H.D.M. Westerink ${ }^{4}$, \\ Jan van Herk ${ }^{4}$, and Kees Tuinenbreijer ${ }^{3}$
}

${ }^{1}$ Center for Telematics and Information Technology (CTIT) / Institute for Behavioral Research (IBR), University of Twente,

P.O. box 217, 7500 AE Enschede, The Netherlands

e.1.vandenbroek@utwente.n1

${ }^{2}$ Department of Artificial Intelligence / Nijmegen Institute for Cognition and Information

(NICI), Radboud University Nijmegen,

P.O. Box 9104, 6500 HE Nijmegen, The Netherlands

e.vandenbroek@nici.ru.nl, mhschutastudent.ru.nl

3 Philips Consumer Electronics, The Innovation Laboratories, Glaslaan 2, SFJ 507 Eindhoven, The Netherlands

\{marleen.schut, kees.tuinenbreijer\}@philips.com

${ }^{4}$ Philips Research, High Tech Campus 34,

5656 AE Eindhoven, The Netherlands

\{joyce.westerink, jan.van.herk\}@philips.com

\begin{abstract}
To improve human-computer interaction (HCI), computers need to recognize and respond properly to their user's emotional state. This is a fundamental application of affective computing, which relates to, arises from, or deliberately influences emotion. As a first step to a system that recognizes emotions of individual users, this research focuses on how emotional experiences are expressed in six parameters (i.e., mean, absolute deviation, standard deviation, variance, skewness, and kurtosis) of physiological measurements of three electromyography signals: frontalis (EMG1), corrugator supercilii (EMG2), and zygomaticus major (EMG3). The 24 participants were asked to watch film scenes of 120 seconds, which they rated afterward. These ratings enabled us to distinguish four categories of emotions: negative, positive, mixed, and neutral. The skewness of the EMG2 and four parameters of EMG3, discriminate between the four emotion categories. This, despite the coarse time windows that were used. Moreover, rapid processing of the signals proved to be possible. This enables tailored HCI facilitated by an emotional awareness of systems.
\end{abstract}

\section{Introduction}

Computers are experienced by their users as coldhearted; i.e., "marked by lack of sympathy, interest, or sensitivity" [1]. However, "during the past decade rapid advances in spoken language technology, natural language processing, dialog modeling, multimodal interfaces, animated character design, and mobile applications all have stimulated interest in a new class of conversational interfaces" [2]. The progress made in this broad range of research and technology enables the rapid computation and modeling of 
empathy for human-computer interaction (HCI) purposes. The latter is of importance since conversation is, apart from being an information exchange, a social activity, which is inherently enforcing [2]. Futurists envision embodied, social artificial systems that interact in a natural manner with us. Such systems need to sense its user's emotional state and should be able to express emotions as well.

Empathic artificial systems can, for example, prevent user frustration in HCI. Users feel frequently frustrated by various causes; i.e., error messages, timed out / dropped / refused connections, freezes, long download time, and missing / hard-to-find features [3]. Picard [4] posed the prevention of user frustration as one of the main goals in HCI. When prevention is not sufficient, online reduction of frustration is needed. According to Hone, Akhtar, and Saffu [5], an (embodied) affective agent, using techniques of active listening and empathy could reduce user frustration.

The analysis of psychophysiological signals is also of use for the validation of computer vision systems that aim to recognize emotional expressions. Then, the latter systems' functioning can be validated and optimized. Knowledge gained from studies on the expression of emotions can also be used for embodied agents, which can, consequently, express emotions in a more realistic manner [6].

The current paper discusses the emotion and their expression through psychophysiological measures, in Section 2 and Section 3 . Next, in Section 4 affective wearables are introduced in which the proposed apparatus for the measurement of the psychophysiological signals can be embedded. Next, the setup of the experiment that is conducted is described in Section 5 , followed by a reduction of the data, in Section 6 , The results are described in Section 7 The paper ends with Section 8 in which the results are discussed, limitations are denoted, and future research is described.

\section{Emotion}

Despite the complexity of the concept emotion, most researchers agree that emotions are acute, intentional states that exist in a relatively short period of time and are related to a particular event, object, or action [7, 8]. In relation with physiology, emotions are predominantly described as points in a two dimensional space of affective valence and arousal, in which valence represents overall pleasantness of emotional experiences ranging from negative to positive, while arousal represents the intensity level of emotion, ranging from calm to excited [9, 10].

The simplest differentiation of emotions is a differentiation between positive and negative emotions. In most cases of HCI, this is sufficient to improve the dialog between user and computer; e.g., when a user has a negative emotion, the computer can adapt its dialog to that, depending on the context. The valence-arousal model differentiates between emotions on both valence (positive and negative affect) and arousal (intensity of the emotion), which allows us to tell the difference between four rough categories of emotions, when differentiated between high valence and low valence and high arousal and low arousal. Some researchers even differentiated between nine categories by including a neutral section on both the valence and arousal axis. However, an, in principle, infinite amount of other arbitrary number of categories can be defined, where the valence and arousal axis not necessarily are divided with the same precision [11]. 
With the determination of emotions, using the valence-arousal model, two difficulties occur: the problem of the lack of extreme coordinates in two categories of the valence-arousal model and the category of emotions, which is called mixed emotions. The first difficulty is mentioned by Lang, Bradley, and Cuthbert [12]. They generated a database of affective pictures (the International Affective Picture System; IAPS). All IAPS pictures were judged according to the valence and arousal dimensions, which resulted in a boomerang shaped figure, with its two extremes in the quadrants high valence - high arousal, and low valence -high arousal. Despite their great efforts, Lang, Bradley, and Cuthbert [12] failed to find pictures that represent the extremities of all four categories.

The second difficulty, mixed emotions, occur when an event, object, or action triggers more than one emotion; e.g., a person feels happy because he sees his old aunt again after many years, but sad because his aunt does not seem to be very healthy anymore. The valence-arousal model, as a two-dimensional space, cannot handle this kind of data; however, the valence-arousal model might be capable of coping with mixed emotions; Konijn and Hoorn [13] suggest that in order to cope with mixed emotions, the valence axis should be unipolar instead of bipolar. They referred to earlier research, which showed that reverse affects do not necessarily have strong negative correlations. When valence is rated on two scales, one for the intensity of positive affect and one for the intensity of negative affect, mixed emotions, in the sense of both positive and negative emotions, will show. As an extension to the valence-arousal model, a unipolar valence axis, with separated positive and negative axes, might allow for a better discrimination between different emotions.

In the current research, solely the valence axis was explored. The reason for this, in addition to the afore mentioned problems of the valence-arousal model, is twofold: 1 ) As a first step to recognize emotions accurately in a long time window (120 sec.), it is necessary to start with simple categories, and 2) Valence has been proved to influence EMG [14].

\section{Psychophysiological Measures}

The research area that considers the effect of psychological processes on physiology is called psychophysiology. The roots of the first psychophysiological area emotions lay in Darwin's book "The expression of emotions in man and animals", which he wrote in 1872. From then on research in psychophysiology has known periods of extensive popularity and even so periods of unpopularity or even rejection. After a period of the latter category, psychophysiology gains interest again due to affective computing.

The overall assumption is that emotion arouses the autonomic nervous system (ANS), which alters the physiological state. This is expressed in various physiological measures; e.g., heart rate, blood pressure, respiration rate, galvanic skin response [15]. In addition, Alpha and Beta brain waves are used to access human attention and emotion [16]. The main advantage of using autonomic physiological measures is that autonomic variables are regulated by the ANS, which controls functions outside the individual's conscious control [15]. 


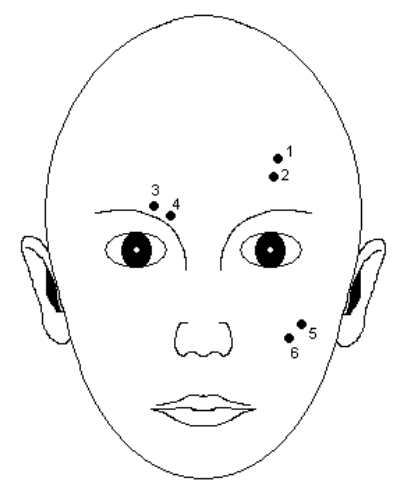

Fig. 1. The points the electrodes that were placed on the face of the participants to determine the EMG signals. The EMG signals of the frontalis, corrugator supercilii, and zygomaticus major were respectively measured through electrodes 1-2, 3-4, and 5-6.

In this research, we focused on how emotional experiences, rated to their positive and negative affect, are expressed in three electromyography (EMG) signals: frontalis, corrugator supercilii, and zygomaticus major (see also Figure 1). The choice of these measures is twofold: 1) a great deal of emotional expression is located in the face [14], as can be measured using facial EMG and 2) the EMG signals can be used to validate computer vision algorithms that aim to detect the emotional expression of people.

EMG measures muscle activity of a certain muscle. Facial EMG is related to affective valence; however, the type of relation depends strongly on the muscle that is measured. The corrugator supercilii, which causes a frown when activated, increases linearly with a decrease in valence, while the zygomaticus major, which is responsible for smiling when activated, increases with an increase in valence [12].

In the Section 5, we will describe the experiment in which the above introduced psychophysiological measures are used in order to verify whether or not they express the emotional state of humans. However, first we will introduce affective wearables in the next section. Such pervasive technology facilitates a system in monitoring its user in an unobtrusive manner. This enables the system to conduct affective computing, which will facilitate in efficient and as pleasant experienced HCI.

\section{Affective Wearables}

Direct physiological measures are often considered to be obtrusive to the user; this is not necessarily true. In the field of affective computing, some efforts have been made to design unobtrusive measurement technology: affective wearables. Picard and Healy [8], define an affective wearable as "a wearable system equipped with sensors and tools which enables recognition of its wearer's affective patterns".

Affective wearables become smaller in time, due to improved design and smaller technology components. Especially when hidden in daily used tools and objects, 
affective wearables could make a huge difference in user acceptance of direct physiological measures.

The acceptance of direct physiological measurements is of great importance since indirect physiological measurement are much more subject to noise. Indirect physiological measurements (e.g., through voice analysis [17]) have been applied in controlled settings such as telepsychiatry [18] and evaluation of therapy effectiveness [17]. However, outside such controlled conditions these measures have not proved to be reliable.

Measurement of physiological signals have already been embedded into wearable tools; e.g., Picard and Scheirer [19] designed the 'Galvactivator', a glove that detects the skin conductivity and maps its values into a led display. In an overview of previous work of the Affective Computing Research Group at MIT, Picard [20] describes several affective wearables. One affective wearable that is of interest in this research is the expression glasses. The expression glasses sense facial movements, which are recognized as affective patterns.

\section{Method}

\subsection{Subjects}

24 Subjects (20 female) were invited from a volunteers database. They signed an informed consent form, and were awarded with a small incentive for their participation. They were aged between 27 and 59 years (average 43 years).

\subsection{Materials}

Sixteen film sequences were selected for their emotional content. Several of these sequences were described by Gross and Levenson [21] for their capability of eliciting one unique emotion among various viewers. They were edited with Dutch subtitles, as is normal on Dutch TV and in Dutch cinemas. Since not enough material of Gross and Levenson [21] was available with Dutch subtitles in acceptable quality, the set was completed with a number of similar sequences. The resulting video fragments each lasted between 9 seconds and 4 minutes. If the fragment lasted less than 120 sec., a plain blue screen was added to make a total of $120 \mathrm{sec}$.

The film fragments were presented on a large 42" $16: 9$ flat panel screen mounted on the wall of the room. Print-outs for significant scenes of each of the film fragments were used to jog the subjects memory of each film fragment after the viewing session.

The psychophysiological measurements were performed with a TMS Portilab system connected to a computer. A ground electrode was attached to the right-hand lower chest area.

Three EMG measurements were done: at the left-hand corrugator supercilii muscle, the right-hand zygomaticus major muscle and the frontalis muscle above the left eye. At each site 2 electrodes were placed in the direction of the muscle (see Figure 1). These signals were first high pass filtered at $20 \mathrm{~Hz}$ and then the absolute difference of the two electrodes was average filtered with a time constant of $0.2 \mathrm{sec}$. 


\subsection{Procedure}

At the beginning of the session, the subject was invited to take place in a comfortable chair and the electrodes were positioned: first at the chest, then at the fingers and then at the face. Then, the recording equipment was checked and aligned when needed. A rest period of 5 minutes was taken into account The subjects were presented with the 16 video fragments, each segment was presented only once.

A pseudo-random order of presentation was generated for the 16 video presentations. This order was designed to spread positive and negative scenes evenly over the session. It was presented to 12 subjects, each starting with a different scene in the list, but maintaining the same order. The reverse order was presented to the other 12 subjects, again each starting with a different scene while maintaining the same presentation order. In between two fragments a plain blue screen was presented for 120 seconds.

After the measuring session, the electrodes were detached, and the subject was requested to fill out a short questionnaire. In this questionnaire, representative pictures of the 16 video fragments were represented sequentially, and the subject was requested to rate them according to various emotion-related axes; e.g., intensity of positive feelings when watching the fragment, and the same for negative feelings.

\section{Data Reduction}

Average intensities for both positive and negative ratings were calculated for each of the film fragments, allowing for a classification of the fragments in 4 emotion categories:

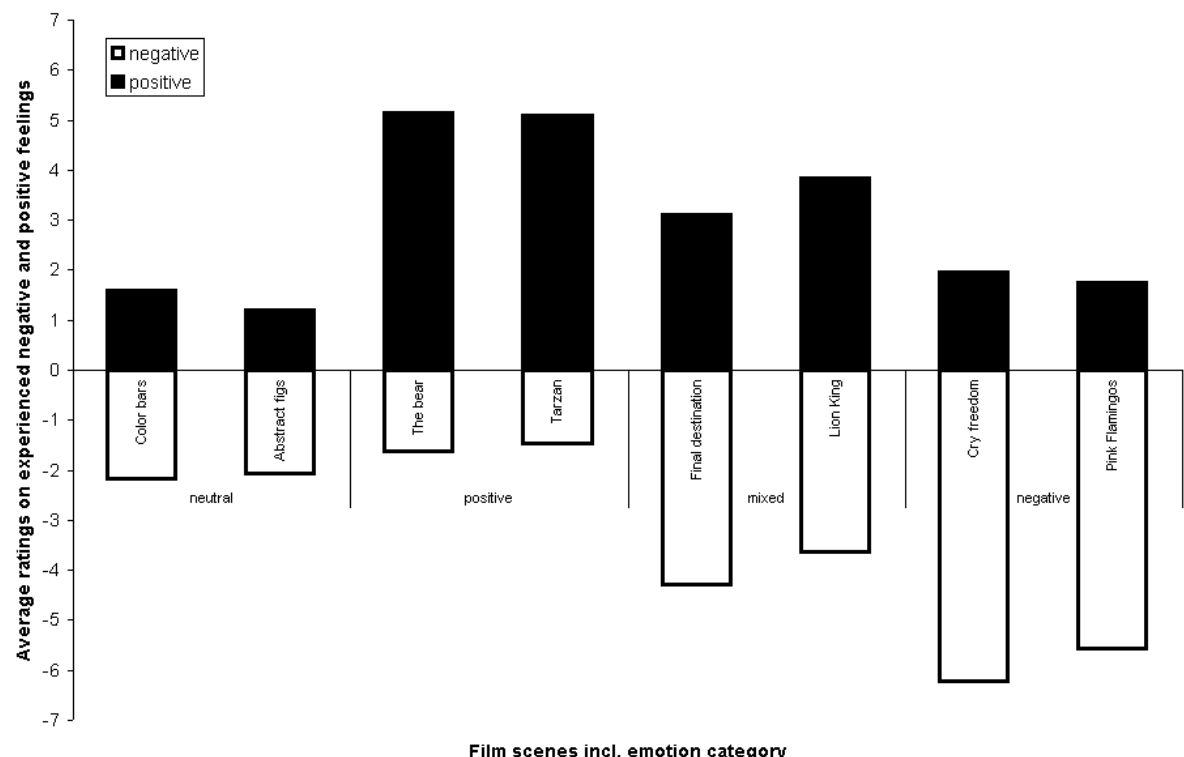

Fig. 2. The eight film scenes with the average ratings given by subjects on both experienced negative and positive feelings. These ratings categorized the film scened into four emotion categories: neutral, mixed, positive, and negative. 
neutral, mixed, positive, negative. In each emotion category, the two fragments with a duration closest to 120 seconds were selected for further analysis (see Figure 2 and Table 1). Furthermore, the EMG data of two subjects appeared to be corrupt; therefore, these datasets were not analyzed.

The three EMG (of the frontalis, corrugator supercilii, and zygomaticus major) signals were measured in order to determine their discriminating ability on the four emotion categories induced by the eight films. To determine the latter, six parameters (mean, absolute deviation, standard deviation, variance, skewness, and kurtosis) were derived from the four signals.

Where the mean, standard deviation, and average deviation are well-known dimensional quantities (i.e., have the same units as the measured quantities $x_{j}$ ), the skewness and kurtosis are conventionally defined as non-dimensional quantities. Both skewness and kurtosis are less well known statistical measures and both are defined in several ways [22, 23]. Therefore, we provide the definitions as adopted in the current research.

The skewness characterizes the degree of asymmetry of a distribution around its mean. It characterizes the shape of the distribution. The usual definition is [22, 23, 24]:

$$
\operatorname{Skewness}\left(x_{1} \ldots x_{N}\right)=\frac{1}{N} \sum_{j=1}^{N}\left[\frac{x_{j}-\bar{x}}{\sigma}\right]^{3}
$$

where $\sigma=\sigma\left(x_{1} \ldots x_{N}\right)$ is the distribution's standard deviation. A positive value of skewness signifies a distribution with an asymmetric tail extending out towards more positive $x$; a negative value signifies a distribution whose tail extends out towards more negative $x$.

Kurtosis measures the relative peakedness or flatness of a distribution relative to a normal distribution. We applied kurtosis as [22, 23, 24]:

$$
\operatorname{Kurtosis}\left(x_{1} \ldots x_{N}\right)=\left\{\frac{1}{N} \sum_{j=1}^{N}\left[\frac{x_{j}-\bar{x}}{\sigma}\right]^{4}\right\}-3
$$

where the -3 term makes the value zero for a normal distribution.

The skewness and kurtosis of EMG signals have been topic of research in previous studies. However, the use of skewness and kurtosis as discriminating descriptors is very limited to only a few studies. In 1983, Cacioppo, Marshall-Goodell, and Dorfman [25] analyzed among a number of parameters, the skewness and kurtosis of skeletal muscle patterns, recorded through EMGs. Four years later, a paper of Cacioppo and Dorfman [24] is published that discusses "waveform moment analysis in psychophysiological research" in general.

In 1989, Hess et al. [26] conducted research toward experiencing and showing happy feelings, also using video segments. Hess et al. [26] recorded four facial EMG signals and extracted the mean, variance, skewness, and kurtosis of these signals. The current 
Table 1. The eight film scenes with the average ratings given by subjects on both experienced negative and positive feelings. Based on the latter two dimensions, the four emotion categories: neutral, mixed, positive, and negative are founded.

\begin{tabular}{llll}
\hline Film scene & Positive & Negative & Emotion category \\
\hline Color bars & 1.60 & 2.20 & neutral \\
Abstract figures & 1.20 & 2.10 & \\
The bear & 5.15 & 1.65 & positive \\
Tarzan & 5.10 & 1.50 & \\
Final destination & 3.11 & 4.32 & mixed \\
Lion King & 3.85 & 3.65 & \\
Cry freedom & 1.95 & 6.25 & negative \\
Pink flamingos & 1.75 & 5.60 & \\
\hline Average & 2.96 & 3.41 & \\
\hline
\end{tabular}

research is distinct from that of Hess et al. [26] since it distinguishes four emotion categories instead of the presence or absence of only one.

\section{Results}

For each of the six statistical parameters, the complete EMG signals were processed over 120 seconds, which was the duration of the films; see also the previous section. For each parameter of each physiological measure, a repeated measures ANOVA was conducted, with the four emotions, each measured with two film scenes, as within-subject factors. So, a total of $18(3 \times 6)$ repeated measures ANOVAs were conducted.

The EMG of the frontalis muscle (see Figure 1) did not provide a significant discrimination between the four emotion categories on any of the statistical parameters. Of all physiological measures, the zygomaticus major signal is the most discriminative physiological signal. The mean, absolute deviation, standard deviation and variance calculated over the zygomaticus major EMG signal showed strong significant effects of emotions, as is illustrated in Figure 3. A significant effect did also show in the skewness of the corrugator supercilii EMG signal (see Figure 3). This is in line with results of previous research of Larsen, Norris, and Cacioppo [14], who concluded that valence influences both the corrugator supercilii and the zygomaticus major.

Not all investigated parameters of all EMG signals proved to be equally suited for sensing a human's emotional state. In particular, the $120 \mathrm{sec}$. averaged values of the physiological signals did not yield significant effects of emotion category, in contrast to what is generally reported in literature. One of the reasons might be that we chose not to correct our data for baseline values, as is common in psychophysiological literature. Another factor is that the present analysis was chosen to extend over a relatively long period of time including the beginning of the video fragment in which the targeted emotions were still in the process of getting elicited, which might have diminished the differences between categories of emotions. Nevertheless, even under these demanding analysis conditions, still some of the measures succeed in distinguishing between the respective emotion categories, as is shown in Figure 3 


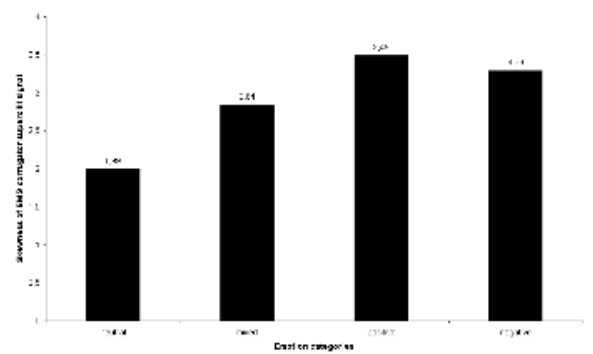

(a)

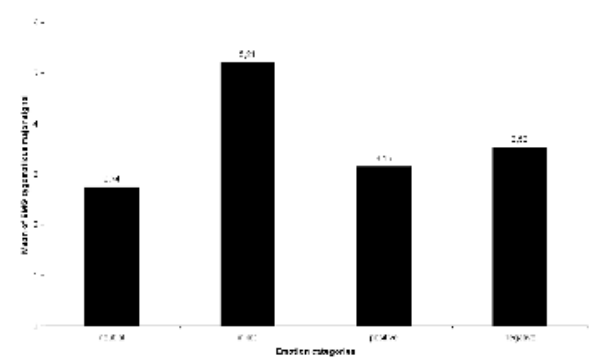

(b)

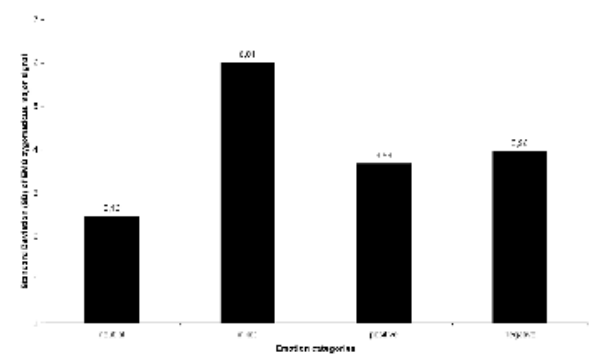

(d)

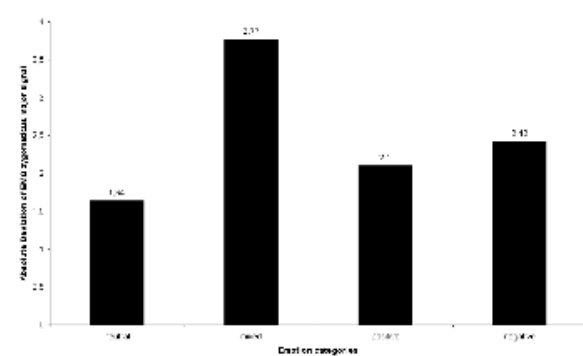

(c)

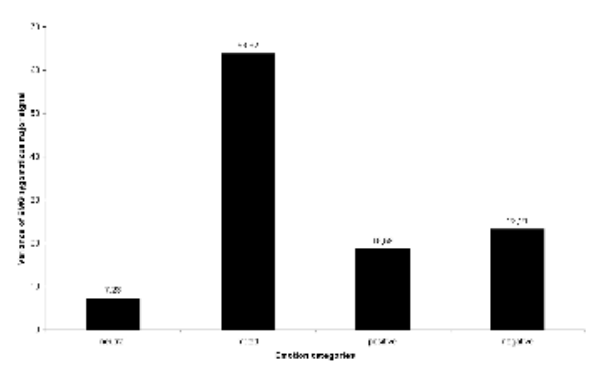

(e)

Fig. 3. The discriminating parameters per emotion category: a) Skewness of EMG corrugator supercilii signal $(\mathrm{F}(3,18)=3.500, \mathrm{p}<0.037)$ and of the EMG zygomaticus major signal the: $\mathrm{b}$ ) mean $(\mathrm{F}(3,18)=9.711, \mathrm{p}<0.001)$, c $)$ absolute deviation $(\mathrm{F}(3,18)=8.369, \mathrm{p}<0.001), \mathrm{d})$ standard deviation $(\mathrm{SD})(\mathrm{F}(3,18)=5.837, \mathrm{p}<0.006)$, and e) Variance $(\mathrm{F}(3,18)=4.064, \mathrm{p}<0.023)$.

\section{Discussion}

Designers envision electronic environments that are sensitive and responsive to the presence and emotion of people. This vision is baptized: "ambient intelligence" and is characterized as embedded, aware, natural, personalized, adaptive, and anticipatory. The set of psychophysiological measures as introduced suits this vision since it can be embedded in wearables, it facilitates awareness for systems connected to it, it aims to mimic human empathy (i.e., is natural), can be connected to a user-profile, senses user's changing emotions, and can facilitate in utilizing knowledge to anticipate on people's emotion and adapt its communication strategy. 
For all four physiological signals used, the parameter skewness proved to be an interesting source of information. The skewness of the distributions of the data of two of the physiological signals differs significantly and for the remaining signal a trend is present, over the four emotions. The skewness characterizes the degree of asymmetry of a distribution around its mean. To inspect the distributions of the signals, more detailed analyzes have to be conducted. Measures such as the peak density should be taken into account for further analysis.

In addition to adding more descriptors of the physiological signals, the time windows of measurement can be changed. In the current setup, the time window enclosed the complete length of the film scene. However, smaller time windows (e.g., 10 or $30 \mathrm{sec}$.) can be applied. Moreover, dynamic time windows can be applied that enclose the time direct after a critical event (if any) appeared in the film scene. The drawback of the latter approach is that it can not be applied in practice, where it can be expected to prove good results for data gathered through experimentation, as in the current research.

A noteworthy observation in the results is that activation of the zygomaticus major EMG shows lower values of the mean, absolute deviation, standard deviation and variance in response to the neutral stimuli than on the other stimuli (see Figure 3). However, a pure neutral stimulus should, in contrast to stimuli of the other emotion categories, not excite a participant at all. In particular in the variance of the zygomaticus major, the difference between neutral emotions and other emotions is shown. This could imply that these statistic values are not only influenced by valence, but by arousal too. Future research should address this issue.

Larsen, Norris and Cacioppo [14] concluded that valence had a stronger effect on the corrugator supercilii than on the zygomaticus major in experiencing standardized affective pictures, sounds, and words, while our research shows a stronger effect of emotions on the zygomaticus major, than on the corrugator supercilii. In addition, the effect is present with four statistical parameters of the zygomaticus major, where it is only present in one statistical parameter (skewness) of the corrugator supercilii.

The difference in strength of the effects found between the current research and that of Larsen, Norris, and Cacioppo [14] can be explained by the fact that other statistical parameters were used in the current research than in that of Larsen, Norris, and Cacioppo [14]. Another difference between the two researches is the type of stimuli. Film scenes are dynamic and multi-modal, they induce emotions by both auditory, and dynamic visual stimuli, as well as affective words, in some fragments. The dynamic and multi-modal characteristics of the film scenes also provide good means to build up emotions, or to create a shock effect. This is not possible with affective words, sounds or pictures of a static character as often used in research to the axes of the valence-arousal model. On the one hand, all these factors give film scenes a relatively high degree of ecological validity [21]. On the other hand, it can not be determined which modality influences the emotional state of the subjects to the highest extent.

A more general notion that can have a significant impact on measurement of emotions is that the emotional state of people changes over time, due to various circumstances. Moreover, different persons have different emotional experiences over the same events, objects, or actions. The latter is determined by a person's personality. Personality traits correlate with affective states, especially with the personality traits extraversion 
and neuroticism, which have been linked both theoretically and empirically to the fundamental affective states of positive and negative affect, respectively [27]. Hence, to enable tailored communication strategies in HCI, not only the emotional state of a person should be determined but also his personality. Providing that the system possesses a personality profile of its user, it will be able to react appropriately to its user's emotions by selecting a suitable communication strategy.

A set of psychophysiological signals is discussed that mirrors one's psychological state up to a high extent; hence, it provides the means to unravel the intriguing feature of humans to sense the emotions of other humans. With that, a first step is made toward a new generation of computer systems. In time, these systems will be able to communicate and debate with us and penetrate our emotional state. Then, computer systems would truly be evolved from coldhearted to warmhearted systems.

\section{References}

1. Merriam-Webster, Incorporated: Merriam-Webster Online. URL: http: / /www.m-w.com/ ([Last accessed on February 6, 2006])

2. Oviatt, S.L., Darves, C., Coulston, R.: Toward adaptive conversational interfaces: Modeling speech convergence with animated personas. ACM Transactions on Computer-Human Interaction 11 (2004) 300-328

3. Ceaparu, I., Lazar, J., Bessiere, K., Robinson, J., Shneiderman, B.: Determining causes and severity of end-user frustration. International Journal of Human-Computer Interaction 17 (2004) 333-356

4. Picard, R.: Affective computing for HCI. In: Proceedings of HCI International (the 8th International Conference on Human-Computer Interaction) on Human-Computer Interaction: Ergonomics and User Interfaces. Volume 1., Lawrence Erlbaum Associates, Inc.: Mahwah, NJ, USA (1999) 829-833

5. Hone, K., Akhtar, F., Saffu, M.: Affective agents to reduce user frustration: the role of agent embodiment. In: Proceedings of Human-Computer Interaction (HCI2003), Bath, UK (2003)

6. Albrecht, I., Schröder, M., Haber, J., Seidel, H.: Mixed feelings: expression of non-basic emotions in a muscle-based talking head. Virtual Reality 8 (2005) 201-212

7. Ortony, A., Clore, G.L., Collins, A.: The cognitive structure of emotions. Cambridge, New York: Cambridge University Press (1988)

8. Picard, R.: Affective Computing. Boston MA.: MIT Press (1997)

9. Ball, G., Breese, J.: Modeling the emotional state of computer users. In: Workshop on Attitude, Personality and Emotions in User-Adapted Interaction, Banff, Canada (1999)

10. Lang, P.J.: The emotion probe: Studies of motivation and attention. American Psychologist 52 (1995) 372-385

11. Bosma, W., André, E.: Exploiting emotions to disambiguate dialogue acts. In: Proceedings of the 9th International Conference on Intelligent User Interface, Funchal, Madeira, Portugal, ACM Press: New York, NY, USA (2004) 85-92

12. Lang, P.J., Bradley, M.M., Cuthbert, B.N.: motion, motivation, and anxiety: Brain mechanisms and psychophysiology. Biological Psychiatry 44 (1998) 1248-1263

13. Konijn, E.A., Hoorn, J.F.: Some like it bad. Testing a model for perceiving and experiencing fictional characters. Media Psychology 7 (2005) 107-144

14. Larsen, J.T., Norris, C.J., Cacioppo, J.T.: Effects of positive and negative affect on electromyographic activity over zygomaticus major and corrugator supercilii. Psychophysiology 40 (2003) 776-785 
15. Scerbo, M.W., Freeman, F.G., Mikulka, P.J., Parasuraman, R., Di Nocero, F.: The efficacy of psychophysiological measures for implementing adaptive technology. Technical Report NASA / TP-2001-211018, NASA Center for AeroSpace Information (CASI) (2001)

16. Aizawa, K., Ishijima, K., Shiina, M.: Summarizing wearable video. In: IEEE International Conference on Image Processing (ICIP). Volume 3., Thessaloniki, Greece (2001) 398-401

17. Van den Broek, E.L.: Emotional Prosody Measurement (EPM): A voice-based evaluation method for psychological therapy effectiveness. Studies in Health Technology and Informatics (Medical and Care Compunetics 1) 103 (2004) 118-125

18. Hilty, D.M., Marks, S.L., Urness, D., Yellowlees, P.M., Nesbitt, T.S.: Clinical and educational telepsychiatry applications: A review. The Canadian Journal of Psychiatry 49 (2004) $12-23$

19. Picard., R.W., Scheirer, J.: The galvactivator: A glove that senses and communicates skin conductivity. In: Proceedings of the 9th International Conference on Human-Computer Interaction, New Orleans (2001)

20. Picard, R.W.: Toward computers that recognize and respond to user emotion. IBM Systems Journal 39 (2000) 705-719

21. Gross, J.J., Levenson, R.W.: Emotion elicitation using films. Cognition and Emotion 9 (1995) 87-108

22. Press, W.H., Flannery, B.P., Teukolsky, S.A., Vetterling, W.T.: Numerical recipes in C: The art of scientific computing. 2nd edn. Cambridge, England: Cambridge University Press (1992)

23. Weisstein, E.W.: CRC Concise Encyclopedia of Mathematics. 2nd edn. Chapman \& Hall/CRC: USA (2002)

24. Cacioppo, J.T., Dorfman, D.D.: Waveform movement analysis in psychophysiological research. Psychological Bulletin 102 (1987) 421-438

25. Cacioppo, J.T., Marshall-Goodell, B., Dorfman, D.D.: Skeletal muscular patterning: Topographical analysis of the integrated electromyogram. Psychophysiology 20 (1983) 269-283

26. Hess, U., Kappas, A., McHugo, G.J., Kleck, R.E., Lanzetta, J.T.: An analysis of the encoding and decoding of spontaneous and posed smiles: The use of facial electromyography. Journal of Nonverbal Behavior 13 (1989) 121-137

27. Matzler, K., Faullant, R., Renzl, B., Leiter, V.: The relationship between personality traits (extraversion and neuroticism), emotions and customer self-satisfaction. Innovative Marketing 1 (2005) 32-39 